\title{
VPMS J1342+2840 - an unusual quasar from the variability and proper motion survey
}

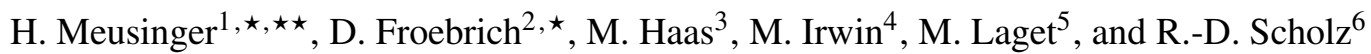 \\ 1 Thüringer Landessternwarte Tautenburg, 07778 Tautenburg, Germany \\ e-mail: meus@tls-tautenburg.de \\ 2 Dublin Institute for Advanced Studies, 5 Merrion Square, Dublin 2, Ireland \\ 3 Astronomisches Institut, Ruhr-Universität Bochum, Universitätsstr. 150/NA7, 44780 Bochum, Germany \\ ${ }^{4}$ Institute of Astronomy, Madingley Road, Cambridge CB3 1HA, UK \\ ${ }^{5}$ Laboratoire d'Astrophysique de Marseille, BP 8, 13376 Marseille Cedex 12, France \\ 6 Astrophysikalisches Institut Potsdam, An der Sternwarte 16, 14482 Potsdam, Germany
}

Received 23 December 2004 / Accepted 16 February 2005

\begin{abstract}
We report the discovery of the highly peculiar, radio-loud quasar VPMS J1342+2840 $(z \approx 1.3)$ from the variability and proper motion survey. We present spectroscopic, imaging and photometric observations. The unusual spectrum shows a strong depression of the continuum over a wide wavelength range in the blue part without the typical structures of broad absorption line (BAL) troughs. The image of the quasar is unresolved and there is no evidence for a foreground object on the line of sight. The broad-band spectral energy distribution is not consistent with obvious dust reddening with the standard SMC extinction curve. The downturn of the continuum flux of VPMS J1342+2840 at short wavelengths can be caused by dust reddening only if the reddening curve is steeper then the SMC curve in the ultraviolet and is very flat at longer wavelengths. Alternatively, the dominant spectral features can be explained by low-ionization BALs forming unusually wide, overlapping absorption troughs.
\end{abstract}

Key words. quasars: absorption lines - quasars: individual: VPMS J1342+2840

\section{Introduction}

An important class of active galactic nuclei are quasars with broad absorption lines (BAL), usually subdivided into lowand high-ionization (LoBAL, HiBAL) quasars. BAL quasars constitute about $15 \%$ of the quasar population (Reichard et al. 2003) and only about $10 \%$ of the BAL quasars are LoBALs. A rare subclass of the LoBALs are the iron LoBALs (FeLoBALs) with absorption from metastable excited states of Fe II and Fe III (Hazard et al. 1987). BALs are naturally explained by powerful, non-isotropic, subrelativistic outflows (Weymann et al. 1991), possibly related to disk winds (Murray \& Chiang 1998). LoBALs probably have higher gas column densities and at least some LoBAL quasars are thus expected to represent either young systems expelling a thick shroud of gas and dust (Voit et al. 1993; Canalizo \& Stockton 2001) or a transition stage between radio-loud and radio-quiet BAL quasars (Becker et al. 1997).

* Visiting Astronomer, German-Spanish Astronomical Centre, Calar Alto, operated by the Max-Planck-Institute for Astronomy, Heidelberg, jointly with the Spanish National Commission for Astronomy.

$\star \star$ Visiting astronomer, Italian Telescopio Nazionale Galileo (TNG) operated at the Spanish Observatorio del Roque de los Muchachos of the Instituto de Astrofisica de Canarias.
Quasars with unconventional spectra are obviously overlooked in quasar surveys based on optical/UV colour or objective prism selection. They are also under-represented in radio surveys with high flux density limits because only a small percentage seems to be radio-loud. Unusual BAL quasars from the Sloan Digital Sky Survey (SDSS) First Data Release were analyzed in detail by Hall et al. (2002; hereafter H02) and the most unconventional quasars from the SDSS Second Data Release were presented by Hall et al. (2004a). Menou et al. (2001) discuss SDSS BAL quasars with radio detection in the VLA FIRST survey. Other quasars with unconventional spectra, e.g. unusual reddening in the UV (Hines 2001) or without obvious metal-line emission (Hall et al. 2004b), have been the subject of a variety of studies. The data base is clearly growing, nevertheless every single odd quasar is an interesting object that may hold clues to various open questions of the structure and the evolution of quasars. Here, we present one of the most unusual quasars from the variability and proper motion survey (VPMS). The VPMS is an optical quasar search project based upon long-term variability and zero proper motion (Scholz et al. 1997; Meusinger et al. 2002, 2003). The quasar selection does not explicitly make use of assumptions on the spectral energy distribution (SED) of quasars and thus the VPMS is expected to be less biased against quasars with unusual SEDs than more conventional optical quasar surveys. 
Table 1. Summary of spectroscopic observations. Column 4 gives the resolution expressed by $F W H M$.

\begin{tabular}{cccccc}
\hline \hline Instrument & Grism & $\begin{array}{c}\lambda \text { range } \\
(\AA)\end{array}$ & $\begin{array}{c}F W H M \\
(\AA)\end{array}$ & $\begin{array}{c}t_{\exp } \\
(\mathrm{ks})\end{array}$ & $\begin{array}{c}\text { Epoch } \\
(\text { year })\end{array}$ \\
\hline CAFOS & B400 & $3600-8000$ & 34 & 3.9 & 2003.3 \\
CAFOS & R200 & $6300-11000$ & 19 & 1.2 & 2003.6 \\
CAFOS & G200 & $5200-9000$ & 15 & 5.4 & 2004.6 \\
DOLORES & LR-B & $3600-8000$ & 22 & 1.8 & 2004.7 \\
DOLORES & LR-R & $5200-9000$ & 15 & 1.2 & 2004.7 \\
\hline
\end{tabular}

\section{Spectroscopy and imaging}

VPMS J1342+2840 $\left(13^{\mathrm{h}} 42^{\mathrm{m}} 46^{\mathrm{s}} .25+28^{\circ} 40^{\prime} 27^{\prime \prime} .5\right.$, J2000.0 $)$ is a faint $(\bar{B}=20.5)$ VPMS quasar, discovered during regular spectroscopic follow-up observations of high-priority quasar candidates in spring 2003. Subsequently, several spectra of moderately higher resolution were taken with CAFOS at the $2.2 \mathrm{~m}$ telescope on Calar Alto, Spain, and with DOLORES at the $3.6 \mathrm{~m}$ Telescopio Nazionale Galileo (TNG) on the island of La Palma, Spain, respectively (Table 1). All observations were done in good atmospheric conditions. The spectra were reduced using the optimal extraction algorithm of Horne (1986).

With its strong depression of the continuum over the whole wavelength interval shortward of $6500 \AA$ (Fig. 1a), the spectrum of VPMS J1342+2840 is remarkably different from the typical quasar spectrum (see Fig. 3a). It is tempting to interpret this depression as due to strong absorption from a blend of iron lines in the spectral region $\lambda \approx 2300-3050 \AA$ and to identify the bump at $\lambda 4400 \AA$ with emission from C III] $\lambda 1909$ corresponding to an (uncertain) systemic redshift of $z_{\mathrm{em}} \approx 1.288$ (i.e., $M_{\mathrm{B}} \approx-27$, after correction for absorption). This interpretation is supported by the identification of several narrow absorption lines (Fig. 1b) at $z_{\text {abs }}=$ 1.2535, most notably Al III $\lambda$ 1860, Ni II(UV12, UV13) $\lambda 2225$, Fe II(UV1) $\lambda 2600$, Fe II(UV62, UV63) $\lambda 2750, \operatorname{Mg}$ II $\lambda 2800$, $\operatorname{Mg} I \lambda 2853, \mathrm{Fe}$ II(UV61) $\lambda 2880$. The Fe II $\lambda 2750$ line seems to be unphysically strong compared to the iron lines at 2400 and $2600 \AA$. The comparison with the CAFOS G200 spectrum shows that the line is possibly slightly enhanced by noise in the TNG spectrum. Nevertheless, the detection of Fe II lines is beyond doubt. There might be also other absorption line systems at slightly different redshifts, yet evidence for them is not as clear as for the $z_{\mathrm{abs}}=1.2535$ system. The abrupt drop-off in flux near Mg II $\lambda 2800$ is of course not explained by one narrow-line system. Such a drop-off is known from several FeLoBAL quasars. However, there are no obvious BAL troughs in VPMS J1342+2840. In this respect, VPMS J1342+2840 resembles SDSS J0105-0033 and SDSS J2204+0031, which were classified by H02 as "mysterious objects".

Unusual spectral properties can be the result of a positional coincidence with a foreground object in combination with gravitational lensing (Irwin et al. 1998; Chartas 2000). A deep $\left(R_{\text {lim }} \approx 23\right) R$ band image taken with CAFOS (Fig. 2) reveals several faint galaxies within $\sim 1^{\prime}$ and faint, fuzzy structures within $\sim 10^{\prime \prime}$. Granted that the faintest structures are associated with the quasar environment, their absolute magnitudes would be $M_{\mathrm{R}} \approx-24.5$, i.e. $\sim 2 \ldots 3.5$ mag brighter than
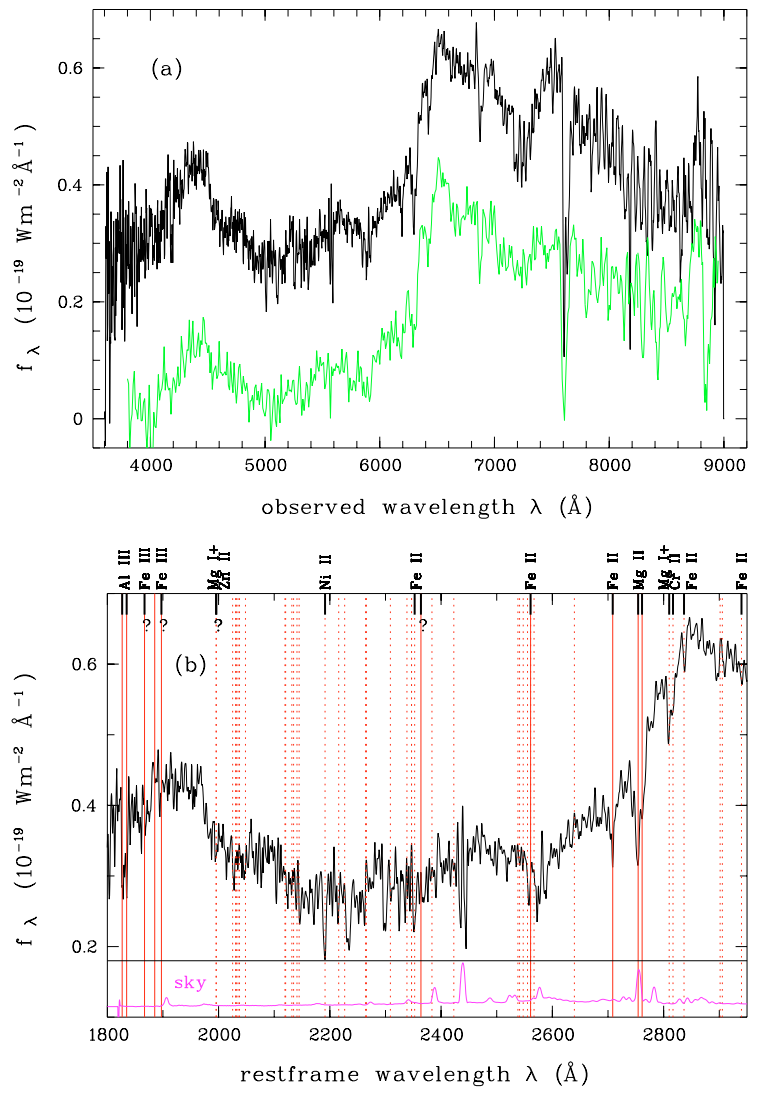

Fig. 1. a) TNG spectrum in the observer frame (dark curve). For comparison, the lower-resolution CAFOS (B400+R200) spectrum is also shown, shifted arbitrarily downward for lucidity. The spectra are not corrected for telluric absorption lines. b) The region between 1800 and $3000 \AA$ of the TNG spectrum in the quasar emission frame. The CAFOS G200 spectrum is similar but has a lower signal-to-noise and is not shown here. Vertical lines: firmly identified transitions in BAL quasars from $\mathrm{H} 02$ (their Table 1); solid lines for transitions that are common in LoBAL and FeLoBAL quasars, dashed lines for rare or unusual transitions. Identified lines are marked at the top ("?" for uncertain identification). Bottom: night sky spectrum (not to scale).

the Schechter magnitude $M_{\mathrm{R}}^{*}$ (e.g., Gaidos 1997; Chen et al. 2003; Christlein \& Zabludoff 2003; $H_{0}=65 \mathrm{~km} \mathrm{~s}^{-1} \mathrm{Mpc}^{-1}$, $q_{0}=0.5, \Lambda=0$ ). Such bright galaxies are extremely rare and thus we conclude that the "fuzz" is more likely to represent foreground galaxies. For the galaxy G1 we measured a spectroscopic redshift of $z=0.142$. However, there is no indication for foreground absorption at this redshift in the spectrum of VPMS J1342+2840. Furthermore, the image of VPMS J1342+2840 is unresolved; there is no indication for a superposition with another object (see inset of Fig. 2). The faint residuals in the PSF-subtracted image are comparable with the residuals typically found for the PSF-stars. Finally, the photometric variability of VPMS J1342+2840 (see below) is common in quasars but is inexplicable for a lensed background galaxy.

\section{Broad-band spectral energy distribution}

To study the SED over a broader spectral range we consider photometric data from several bands: photometry in the 


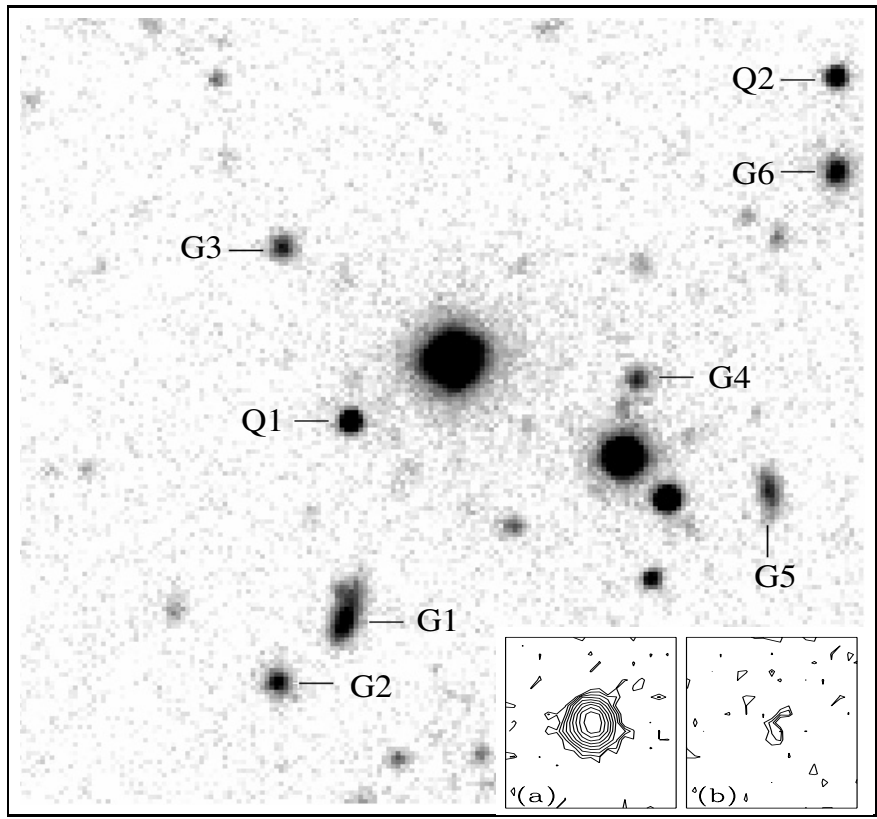

Fig. 2. $90^{\prime \prime} \times 90^{\prime \prime}$ field around VPMS J1342+2840 (Q1) in the $R$ band ( $\mathrm{N}$ is up, $\mathrm{E}$ is left). The other quasar (Q2) in the image is VPMS J1342+2841 $(z=0.873)$. Larger extended objects are labeled with "G". The bright star in the center is GSC2.2 N1301033185 $(R=$ 14.9). Inset: contour plots (equally spaced logarithmic intervals) of the $10^{\prime \prime} \times 10^{\prime \prime}$ area containing a) the original image of VPMS J1342+2840; and $\mathbf{b})$ the residuals after subtraction of the PSF.

$J H K^{\prime}$ bands was performed with MAGIC at the $2.2 \mathrm{~m}$ telescope on Calar Alto. BVR magnitudes are from images taken with CAFOS at the same telescope. The $U$ magnitude comes from the VPMS measurements on Schmidt plates taken later than 1990 (see below). The field has been observed with the balloon-borne ultraviolet imaging telescope FOCA (Laget et al. 1991) at $\lambda 2000 \AA$; the flux limit at half completeness is $0.026 \mathrm{mJy}$. As expected, there is no significant detection of VPMS J1342+2840 since the Lyman limit comes close to the FOCA band for $z \approx 1.3$. Finally, an $I$ magnitude, without error bar, is available from the USNO-B1.0 catalogue (Monet et al. 2003) measured on a POSS II IV-N plate taken in 1997.

The resulting broad-band SED is shown in Fig. 3a. The uncertainty due to variability is expected to be small. However, we caution that changes in flux cannot be ruled out. The $B$ band lightcurve from the VPMS data shows a dimming by $\sim 0.8$ mag between $\sim 1970$ and $\sim 1990$ but only small fluctuations between $\sim 1990$ and 2003. A similar behaviour is indicated by the UVR data. (The dimming is stronger at shorter wavelengths, as is typical for quasars, cf. Trévese et al. 2001.) Figure 3 reflects the depression of the continuum seen in Fig. 1a. We restrict the power-law approximation $f_{v} \propto v^{\alpha}$ to $\lambda>6500 \AA$ (observed) and find $\alpha=-0.80$ from the linear regression of the $K^{\prime} H J R$ magnitudes. If we exclude the $K^{\prime}$ data point (it is not clear whether the turnup at $2.2 \mu \mathrm{m}$ is real), the fit is considerably improved and the slope $\alpha=-0.54$ comes close to the slope of the composite spectrum from all VPMS quasars.

We found a positionally coincident $\left(\sim 1^{\prime \prime}\right)$ radio source on the image cutout from the NRAO Very Large Array FIRST

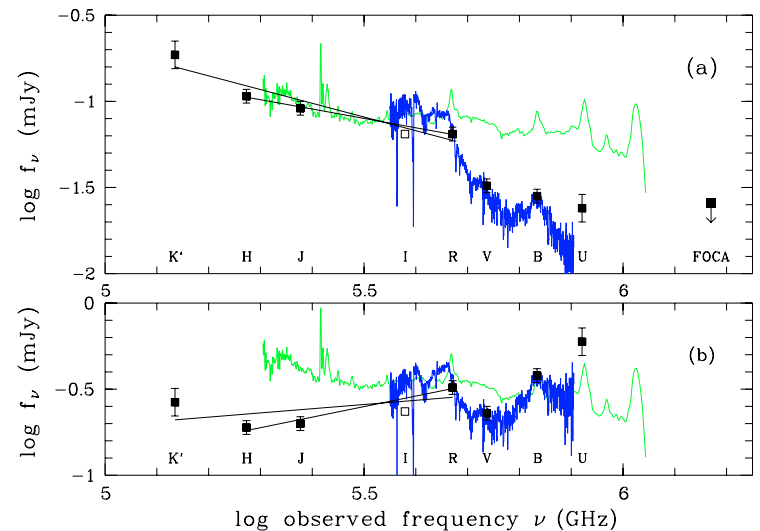

Fig. 3. a) The observed SED from photometric data in various bands (symbols) with linear regression lines (see text). Superimposed are the TNG spectrum from Fig. 1 (dark curve) and the mean VPMS quasar spectrum (thin curve). The fluxes were corrected for galactic foreground absorption using the extinction maps of Schlegel et al. (1998). b) The same after dust reddening correction for the SMC extinction curve from Pei (1992) with $E(B-V)=0.3$.

survey (Becker et al. 1995) with a peak flux density of $0.7 \mathrm{mJy}$ and an integrated flux density of $2.3 \mathrm{mJy}$ at $1.4 \mathrm{GHz}$. The source is not listed in the FIRST catalogues because of the hard detection limit of $1 \mathrm{mJy}$, which applies to the peak flux density rather than to the integrated flux density. In the NRAO 1.4 GHz VLA Sky Survey (NVSS; Condon et al. 1998), a source with a peak flux density of $1.6 \mathrm{mJy}$ is seen at a distance of $\sim 5^{\prime \prime}$. According to NED ${ }^{1}$, VPMS J1342+2840 is not detected at other radio bands; thus the radio spectral index is not available. We use the $\mathrm{K}$-corrected ratio $R^{*}$ of the $5 \mathrm{GHz}$ radio flux density to the $2500 \AA$ optical flux density in the quasar rest frame as a measure of radio-loudness. Using the standard definition with $\alpha_{\text {radio }}=-0.5$ and $\alpha_{\text {opt }}=-1$ (Stocke et al. 1992), we find $\log R^{*}=1.7$. If we consider that (a) the observed flux at $2500 \AA$ (rest frame) is reduced; and (b) $\alpha_{\mathrm{opt}}=-0.54$ or -0.80 (Fig. 3a), we get $\log R^{*}=1.52$ or 1.56 , respectively, for the FIRST radio flux. With $\alpha_{\text {radio }}=-0.3$ (White et al. 2000), the corresponding values are $\log R^{*}=1.55$ and 1.60 . Adopting a divide between radio-quiet and radio-loud quasars at $\log R^{*} \approx 1$ (e.g., Stocke et al. 1992), VPMS J1342+2840 has to be classified as radio-loud.

\section{Discussion}

Is the peculiar spectrum of VPMS J1342 +2840 due to strong dust extinction? The reddening associated with mildly obscured quasars has typically been found to be consistent with that of the Small Magellanic Cloud (SMC; Hopkins et al. 2004). On the other hand, Gaskell et al (2004) argue that most quasars are affected by extinction with a reddening curve which is distinct from the SMC-curve. To de-redden the spectrum of VPMS J1342+2840 we adopt three different reddening curves: (a) SMC-like; (b) relation from Gaskell et al.;

\footnotetext{
1 The NASA/IPAC Extragalactic Database is operated by the Jet Propulsion Laboratory, California Institute of Technology, under contract to NASA.
} 
(c) Galactic curve. The colour excess $E(B-V)$ is used as a free parameter to fit the blue end of the corrected TNG spectrum to the composite spectrum from all VPMS quasars which was normalized before to the red end of the corrected TNG spectrum. For SMC-like reddening, the de-reddened blue $(\lambda \lesssim 2000 \AA)$ end of the corrected continuum matches the mean quasar spectrum for $E(B-V) \approx 0.3$ (Fig. 3b). However, there is a strong discrepancy in the intermediate part of the spectrum (2000 to $2800 \AA$ rest frame). Moreover, the de-reddened broad-band SED has a very untypical shape and does not fit the mean quasar spectrum over a broader spectral range. The fit is even worse for Galactic extinction with its $\lambda 2175 \AA$ bump and it is much worse for the curve from Gaskell et al. which is too flat at short wavelengths. Given that the slope of the red and near-IR part of the uncorrected broad-band SED is well matched by the usual power-law, we conclude that dust can be a major reason for the peculiar spectrum of VPMS J1342+2840 only for an unusual extinction curve which must be very flat at $\lambda \gtrsim 2900 \AA$ and steeply rising at shorter wavelengths. Stronger than SMClike reddening in the UV has been suggested by H02. Hines et al. (2001) argue that the downturn at $\lambda \lesssim 2500 \AA$ in the spectra of two IRAS quasars might be produced by dusty scattering.

Another likely explanation for the depression at $\lambda \lesssim$ $3000 \AA$ (rest frame) are wide, overlapping BAL troughs. Such an interpretation requires absorption that increases in strength when BALs overlap. This problem has been discussed at length by $\mathrm{H} 02$ in the context of the two "mysterious objects" SDSS J0105-0033 and SDSS J2204+0031. These authors argue that barely plausible fits can be achieved with wide troughs detached by $\sim 12000 \mathrm{~km} \mathrm{~s}^{-1}$ if there is partial covering of different regions of the continuum source as a function of velocity. Partial covering seems not implausible for VPMS J1342+2840: it is known that absorption in BAL outflows is saturated, even though the troughs rarely reach zero flux. This nonblack saturation can be due to scattered light that bypasses the absorbing region or due to partial covering of the continuum source (Arav et al. 1999). Because of the strong variability on timescales of a few years it seems unlikely that the observed blue continuum of VPMS J1342+2840 is dominated by scattered light. Following H02, the only object known to definitely exhibit spatially distinct velocitydependent partial covering is FBQS $1408+3054$. If our interpretation is true, VPMS J1342+2840 would be another representative of these extremely rare objects. Moreover, it is tempting to speculate that VPMS J1342+2840 is a "missing link" (perhaps transitional) between FBQS 1408+3054 and the "mysterious" SDSS quasars.

\section{Conclusion}

VPMS J1342+2840 is an unresolved radio-loud quasar at $z \approx$ 1.3 with a very peculiar spectrum. Both the optical spectrum and the broad-band SED are not consistent with obvious reddening by SMC-like dust. Either the reddening curve is (a) much steeper between 2000 and $2900 \AA$; and (b) flat at longer wavelengths, or the absorption comes from unusual BAL features dominated by wide, overlapping troughs with velocity-dependent partial covering of the central source. The dusty scattering hypothesis can be tested by spectropolarimetry (e.g. Hines et al. 2001). With its high variability index VPMS J1342+2840 is one of the highest priority quasar candidates at the faint end of the VPMS. Obviously, the VPMS provides a useful tool for the detection of unconventional quasars.

Acknowledgements. We are grateful to the anonymous referee and to Patrick Hall for helpful comments. This research is based on observations made with the Italian Telescopio Nazionale Galileo (TNG) operated on the island of La Palma by the Centro Galileo Galilei of the INAF (Istituto Nazionale di Astrofisica) at the Spanish Observatorio del Roque de los Muchachos of the Instituto de Astrofisica de Canarias and with the $2.2 \mathrm{~m}$ telescope of the German-Spanish Astronomical Centre, Calar Alto, Spain. We acknowledge the staff members of these telescopes for their kind assistance. H.M. acknowledges financial support from the European Optical Infrared Coordination Network for Astronomy, OPTICON, and from the Deutsche Forschungsgemeinschaft under grants Me1350/17-1 and Me1350/18-1. D.F. received funding by the Cosmo Grid project, funded by the Irish Higher Education Authority. M.H. thanks for grants from the NordrheinWestfälische Akademie der Wissenschaften.

\section{References}

Arav, N., Becker, R. H., Laurent-Muehleisen, S. A., et al. 1999, ApJ, 524, 566

Becker, R. H., White, R. L., \& Helfand, D. J. 1995, ApJ, 450, 559

Becker, R. H., Gregg, M. D., Hook, I. M., et al. 1997, ApJ, 479, L93

Canalizo, G., \& Stockton, A. 2001, ApJ, 555, 719

Chartas, G. 2000, ApJ, 531, 81

Chen, H.-W., Marzke, R., McCarthy, P., et al. 2003, ApJ, 586, 745

Christlein, D., \& Zabludoff, A. 2003, AJ, 591, 764

Condon, J. J., Cotton, W. D., Greisen, E. W., et al. 1998, AJ, 115, 1693

Gaidos, E. J. 1997, AJ, 113, 117

Gaskell, C., Goosmann, R., Antonucci, R., et al. 2004, ApJ, 616, 147

Hall, P., Anderson, S., Strauss, M., et al. 2002, ApJS, 141, 267 (H02)

Hall, P., Knapp, G. R., Richards, G. T., et al. 2004a [arXiv: astro-ph/0403347]

Hall, P., Hoversten E., Tremonti, C., et al. 2004b, AJ, 127, 3146

Hazard, C., McMahon, R. G., Webb, J. K., et al. 1987, ApJ, 323, 263

Hines, D. C., Schmidt, G. D., Gordon, K. D., et al. 2001, ApJ, 563, 512

Hopkins, P. F., Strauss, M. A., Hall, P. B., et al. 2004, AJ, 128, 1112 Horne, K. 1986, PASP, 98, 609

Irwin, M. J., Ibata, R. A., Lewis, G. F., et al. 1998, ApJ, 505, 529

Laget, M., Burgarella, D., Milliard, B., et al. 1992, A\&A, 259, 510

Menou, K., Vanden Berk, D. E., Ivezić, Ž., et al. 2001, ApJ, 561, 645

Meusinger, H., Scholz, R.-D., Irwin, M., et al. 2002, A\&A, 392, 851

Meusinger, H., Brunzendorf, J., \& Laget, M. 2003, Astron. Nachr., 324,474

Monet, D. G., Levine, S. E., Casian, B., et al. 2003, AJ, 125, 984

Murray, N., \& Chiang, J. 1998, ApJ, 494, 125

Pei, Y. C. 1992, ApJ, 395, 130

Reichard, T. A., Richards, G. T., Hall, P. B., et al. 2003, AJ, 126, 2594

Richards, G. T., Hall, P. B., \& Vanden Berk, D. E. 2003, AJ, 126, 1131

Schlegel, D., Finkbeiner, D., \& Davis, M. 1998, ApJ, 500, 525

Scholz, R.-D., Meusinger, H., \& Irwin, M. 1997, A\&A, 325, 457

Stocke, J. T., Morris, S. L., Weymann, R. J., et al. 1992, ApJ, 396, 487

Trévese, D., Kron, R. G., \& Bunone, A. 2001, ApJ, 551, 103

Voit, G. M., Weymann, R. J., \& Korista, K. T. 1993, ApJ, 413, 95

Weymann, R. J., Morris, S. L., Foltz, C. B., et al. 1991, ApJ, 373, 23

White, R. L., Becker, R. H., Gregg, M. D., et al. 2000, ApJS, 126, 133 